\title{
Towards the Nanoscale-ultrathin Metal Coatings as a Solution for Imaging of Fine- scale Structures
}

Anna Walkiewicz

Quorum Technologies, Laughton, England, United Kingdom

The raising amount of nanofibers applications, especially those produced in electrospinning process, creates a need of imaging them to the extent that allows to examine not only their alignment and diameter but also their discrete morphology.

Electrospinning fibers are used in many fields- from energy storage (solar cells, fuel cells), environmental engineering (membranes and filters) to healthcare (tissue engineering and drug delivery). Usually they are processed further through chemical modification that equip their surface with active molecules. Yet, before that can happen, it is essential to examine their native surface to govern the post-treatment process in the desired way.

The fibers could have variety of diameters from hundreds to a few nanometers, where usually, the smallest diameters are in preference. They can be smooth or exhibit porosity.

Close examination of such tiny polymeric structures is usually carried out by use of Scanning Electron Microscopy.

Electrospinning fibers are made of polymers, they form stacks, where individual fibers touch each other but they are not connected. In SEM imaging such samples are the most challenging as fibers gather charge and might move when subjected to the electron beam. For successful SEM imaging of electrospinning fibers a layer of conducting coating is a necessity.

Such coating has to be made of a metal with excellent SE yield that exhibits very small grain size. Only in such case the coating can be thin enough not to obscure the discrete morphology of a single fiber and allow for crisp and clear imaging.

Q150V Plus coater allows to coat electrospinning fibers in such a way that it is possible to image even very small fibers and examine their discrete morphology.

Gold coatings were chosen to present the effect of coating parameters as base vacuum and sputtering current on the quality of SEM imaging. Gold, iridium, molybdenum and tungsten coatings produced with use of high base vacuum were used to show the impact of a metal grain size on coating quality in ultrahigh resolution imaging. PVdF - poly(vinylidene fluoride) electrospinning fibers were chosen as substrates for all metal coatings and subjected to imaging. 


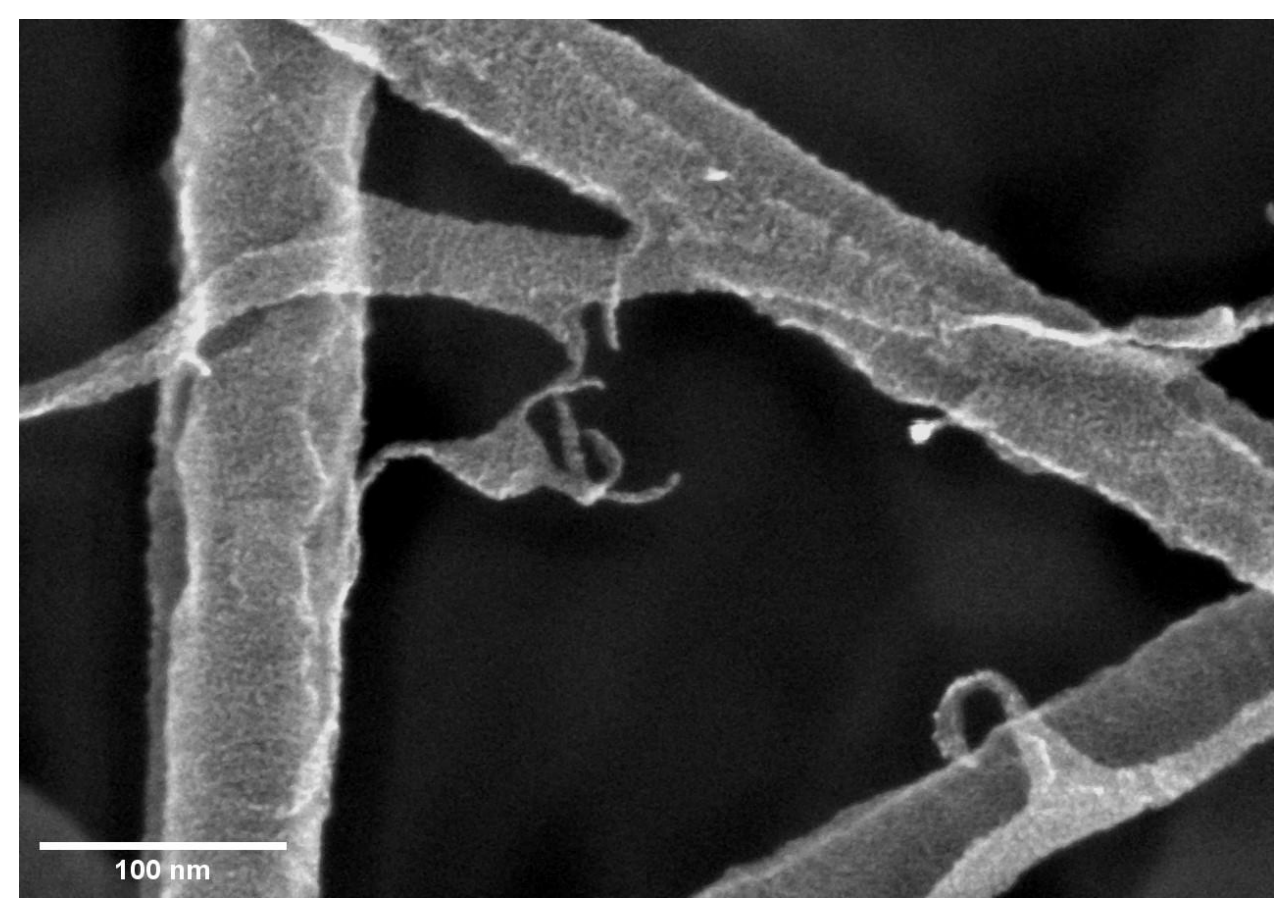

Figure 1. PVdF electrospinning fibers coated with $1 \mathrm{~nm}$ of iridium

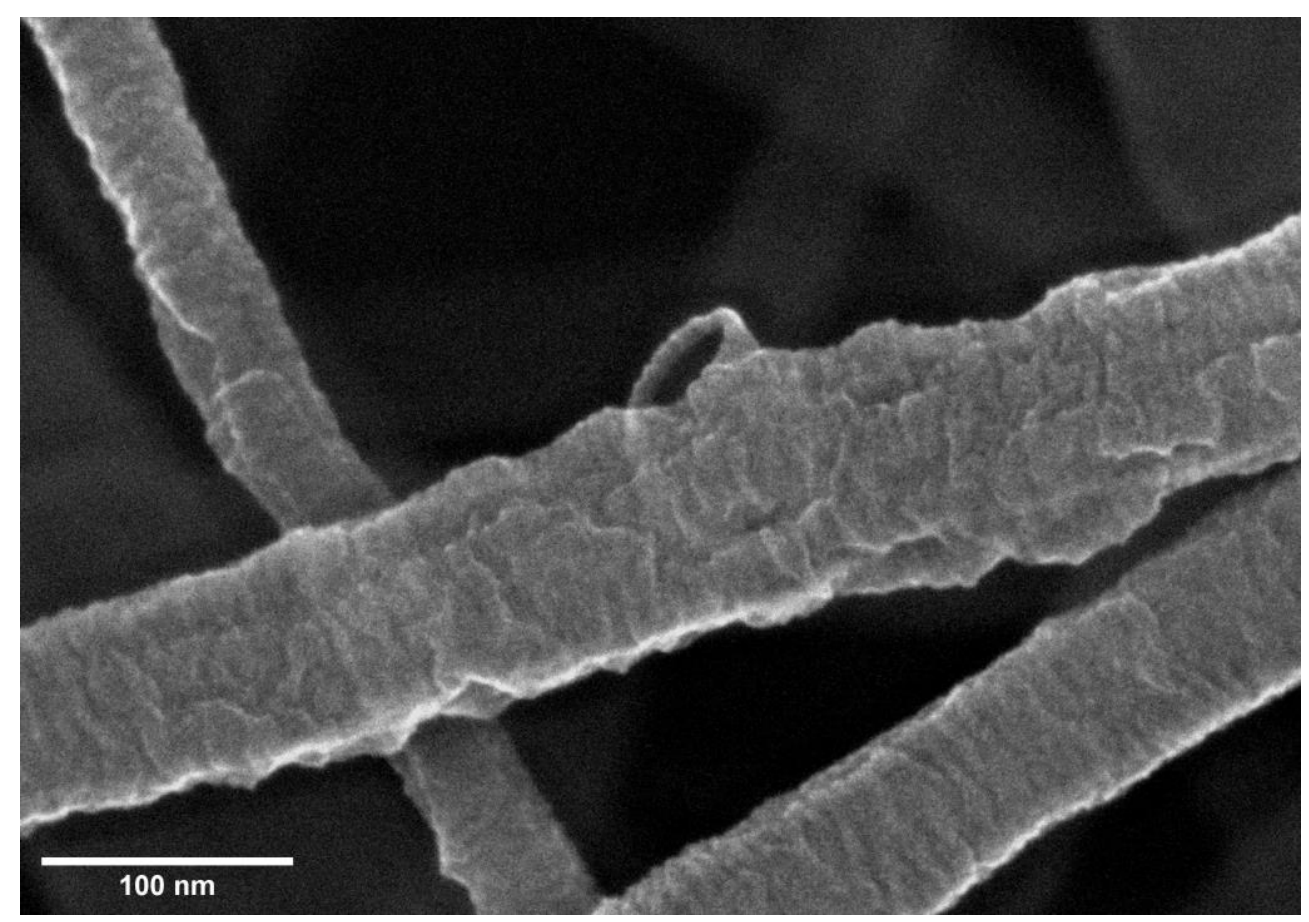

Figure 2. PVdF electrospinning fibers coated with $1 \mathrm{~nm}$ of tungsten

\section{References}

[1] Pham, Q.P.; Mikos, Antonios G. Tissue Engineering, 12, 1197-1211(2006)

[2] Ewaldz, E; Pattel, R; Banerjee, M; Brettmann, B K, Polymer, 153, 529-537(2018)

[3] Li, X.; Cheruvally, G.; Kim, Journal of Power Sources, 167, 491-498 (2007)

[4] Lin, T.; Wang, H.; Wang, H.; Wang, X. Nanotechnology, 15, 1375 (2004) 